\title{
Genetic disorders and disease mechanisms | ABSTRACTS
}

\section{3}

COL7A1 mutations in recessive dystrophic epidermolysis bullosa patients identified by next generation sequencing inherited diseases panel

B Mayer, ${ }^{1} \mathrm{P}$ Sillo, ${ }^{1} \mathrm{~T}$ Szemes, ${ }^{2} \mathrm{M}$ Hýblova, ${ }^{2} \mathrm{D}$ Pinter, ${ }^{1} \mathrm{M}$ Mazan ${ }^{1}$ and S Kárpáti ${ }^{1} 1$ Semmelweis University, Budapest, Hungary and 2 Comenius University, Bratislava, Slovakia Our aim was to identify mutations in the COL7A1 gene in recessive dystrophic epidermolysis bullosa patients and to look for further genetic changes in genes that encode proteins along the basement membrane and could potentially modify disease phenotype interacting with collagen VII. Four patients ( 1 female, 1 year old; 3 males, 8,10 and 54 years old) were diagnosed having severe generalized and localized recessive dystrophic epidermolysis bullosa (RDEB) based on clinical signs, family history (negative) and immunofluorescent antigen mapping of skin biopsies. For genetic studies, genomic DNA was isolated from peripheral blood leukocytes. We used a next generation sequencing panel containing 508 genes including COL7A1 and other epidermolysis bullosa associated genes. The panel was developed to allow detection of the genetic background of early onset severe childhood recessive disorders. Sequence data were evaluated using Genetalk and HGMD database. Mutations obtained from next generation screening, were confirmed with Sanger sequencing. We found known homozygote COL7A1 (c.425A>G, p.K142R) mutations that are prevalent in central Europe in three patients (severe generalized RDEB), known homozygote c.6654C >G, G2218G synonym splice mutations in the same patients and two so far unknown mutations (c.5869C>T, p.R1957W; c.8457T>G, p.Y2819X) in the compound heterozygote adult patient (localized RDEB). On the other hand, no additional candidate mutations were found in epidermolysis bullosa associated genes (LAMB3, LAMC2) and genes (COL1A1, LAMA5) those protein products were described to interact with collagen VII and could potentially influence phenotypes of the disease.

\section{5}

Epidemiologic and genetic association between atopic dermatitis, rheumatoid arthritis, inflammatory bowel disease, and type- 1 diabetes

H Baurecht, ${ }^{1}$ J Schmitt, ${ }^{2}$ E Rodriguez, ${ }^{1}$ W Lieb, ${ }^{6}$ C Gieger, ${ }^{3}$ A Irvine, ${ }^{4}$ N Novak ${ }^{5}$ and S Weidinger 11 Department of Dermatology, Venereology and Allergy, University Hospital Schleswig-Holstein, Kiel, Germany, 2 Center for Evidence-based Healthcare, Medizinische Fakultät Carl Gustav Carus, TU Dresden, Dresden, Germany, 3 Institute of Genetic Epidemiology, Helmholtz Zentrum München, German Research Center for Environmental Health, Neuherberg, Germany, 4 Department of Paediatric Dermatology, Our Lady's Children's Hospital, Crumlin, Dublin, Ireland, 5 Department of Dermatology and Allergy, University of Bonn, Bonn, Germany and 6 Institute of Epidemiology and PopGen Biobank, University Hospital Schleswig-Holstein, Kiel, Germany

Atopic dermatitis (AD) is characterized by epidermal barrier failure and cutaneous inflammation. Molecular studies suggested shared genetic factors and immunological pathways with other inflammatory diseases as rheumatoid arthritis (RA) and inflammatory bowel disease (IBD), but epidemiological evidence is scarce. We test the hypothesis that prevalent $A D$ is a risk factor for incident RA and IBD and inversely related to type-1 diabetes (T1D) and investigate RA, IBD, and T1D susceptibility loci in AD. This cohort study utilized data from German National Health Insurance beneficiaries age 40 or younger $(n=655,815)$ from 2005 through 2011. Prevalent AD in 2005/2006 was defined as primary exposure, and incident RA, IBD, and T1D in 2007-2011 as primary outcomes. Risk ratios were calculated and established RA, IBD and T1D loci were explored in high density genotyping data. Patients with prevalent $A D$ were at increased risk for incident RA (risk ratio (RR) 1.72, $95 \% \mathrm{Cl}=1.25-2.37), \mathrm{CD}(\mathrm{RR} 1.34,95 \% \mathrm{Cl}=1.11-1.61)$ and $\mathrm{UC}(\mathrm{RR} 1.25,95 \% \mathrm{Cl}=1.03-1.53)$. There was no disproportionate occurrence of known RA, CD, UC or T1D risk alleles in AD. AD is a risk factor for the development of RA and IBD. The excess comorbidity cannot be attributed to major known IBD and RA genetic risk factors.

\section{7}

Association of two rare genetic diseases confirmed by next generation sequencing $\underline{\text { P Silloo }},{ }^{1}$ B Mayer, ${ }^{1}$ T Szemes, ${ }^{2}$ M Hýblova, ${ }^{2}$ D Pinter, ${ }^{1}$ M Mazan, ${ }^{1}$ K Nemeth ${ }^{1}$ and S Kárpáti ${ }^{1} 1$ Semmelweis University, Budapest, Hungary and 2 Comenius University, Bratislava, Slovakia Based on the characteristic skin symptoms and the positive family history for a blistering skin disease as well as on the immunofluorescent antigen mapping of the skin, generalized intermediate recessive dystrophic epidermolysis bullosa was diagnosed in a girl short after birth. Because of hyperactivity, attention disorder and learning disabilities she was also investigated for psychiatric and neurologic diseases. Niemann-Pick type $\mathrm{C}$ was first suspected when she also developed action dystonia, cerebellar ataxia, difficulty in swallowing, dysarthria and hepatosplenomegaly. Mutations of both rare genetic diseases were identified by next generation sequencing and a gene panel for early onset recessive disorders. She was compound heterozygote for COL7A1 c.5869C>T, p.R1957W and for c.7012C > T, p.R2338X mutations explaining her recessive dystrophic epidermolysis bullosa. In addition, she carried the compound heterozygous NPC1 c. 1185 1186insT, p. D396*, and c.2861C > T, p.S954L mutations as a genetic background for her Niemann-Pick type C disease symptoms.

\section{4}

Keratitis-Ichthyosis-Deafness Syndrome Associated Mutations Impair the Localization and Functions of Connexin 26

H Aypek and G Mese Izmir Institute of Technology, Izmir, Turkey

Connexins ( $\mathrm{Cx}$ ) form gap junctions and non-junctional hemichannels that play roles in several cellular mechanisms, including proliferation and differentiation. The importance of connexins for human physiology was shown by the association of mutations in several isoforms with various human hereditary disorders. Mutations in Cx26 cause both non-syndromic and syndromic deafness associated with skin disorders including keratitis-ichthyosis-deafness (KID) syndrome. In vitro characterization of Cx26 mutations suggested that mutations causing non-syndromic deafness and syndromic deafness show different properties, where the former ones result in loss-of-function and the latter ones cause gain-of-function mutations. For example, $\mathrm{C} \times 26$ mutations linked to keratitis-ichthyosis-deafness (KID) syndrome were shown to result in the formation of abnormal hemichannels. Here, we assessed the effect of two recently identified Cx26 mutations associated with KID syndrome, 130N and D50Y, on protein biosynthesis and channel function in both communication deficient cell lines and keratinocytes. Immunostaining experiments showed the failure of $\mathrm{I} 30 \mathrm{~N}$ and D50Y to form gap junction plaques at cell-cell contact sites, which is corroborated with an accumulation of mutant proteins in the Golgi apparatus. Fluorescent dye uptake assays revealed an increase in the uptake of neurobiotin and ethidium bromide into cells with I30N and D50Y mutations compared to WT containing cells, indicating the presence of abnormal hemichannels. Further, cells with mutant proteins appeared to have elevated intracellular calcium levels compared to WT transfected cells as measured by Fluo-3AM loading and flow cytometry analysis. In conclusion, I30N and D50Y mutations resulted in the formation of aberrant hemichannels similar to previously characterized KID syndrome mutations. They also caused an increase in intracellular calcium content, a process which may influence various cellular processes that may contribute to the development of epidermal phenotypes of KID syndrome.

\section{6}

\section{WITHDRAWN}

\section{8}

Molecular analysis of antibiotic resistance and virulence features of Staphylococcus sp. strains isolated from patients with chronic leg ulcers

M Georgescu, ${ }^{1}$ I Gheorghe, ${ }^{3}$ CF GHIATAU, ${ }^{2}$ L DASCALU $^{1}$ and CM Chifiriuc ${ }^{1} 1$ Microbiology Department, University of Bucharest, Faculty of Biology, Bucharest, Romania, 2 Dr. Carol Davila Central Military Universitary Emergency Hospital "SUUMC Dr. Carol Davila", Bucharest, Romania and 3 Research Institute of the University of Bucharest - ICUB, Bucharest, Romania

Methicillin-resistant Staphylococcus aureus (MRSA) is one of the most common organisms isolated from clinical samples, being associated with high morbidity and mortality rates among hospitalized patients. The aim of this study was to evaluate the prevalence, antibiotic susceptibility and virulence profiles of methicillin-sensitive and methicillin-resistant Staphycolococcus sp. isolates collected from patients with chronic leg wounds. A total of 22 isolates of Staphylococcus spp. were collected from hospitalized patients with chronic leg wounds. The isolates were identified by using the conventional biochemical tests. We used PCR to determine the presence of the following genes responsible for antibiotic resistance and virulence profiles: mecA, SCC mec V1J, dcs, type IVa, ebps (elastin binding proteins), bbp (bone sialoprotein binding protein), hlg (haemolysin gamma gene). Of 22 isolates, 13 isolates ( $59 \%$ ) were found to be MRSA. Regarding the genes responsible for antibiotic resistance, the PCR analysis revealed: 8 - mecA, 7 - SCC mec V1J, 2 - dcs, 6 - type IVa. The majority of Staphylococcus sp. isolates harbored the investigated virulence genes (hlg -12 , ebps -5, bbp -2 strains). The majority of the Staphylococcus spp. isolates are both virulent and resistant to antibiotics, features which must be taken into account for a proper therapeutic management of chronic wound patients. 